\title{
Phenomenology of $E_{6}$-inspired leptophobic $Z^{\prime}$ boson at the LHC
}

\author{
Cheng-Wei Chiang, ${ }^{a, b, c}$ Takaaki Nomura $^{d}$ and Kei Yagyu ${ }^{a}$ \\ ${ }^{a}$ Department of Physics and Center for Mathematics and Theoretical Physics, \\ National Central University, Chungli, Taiwan 32001, R.O.C. \\ ${ }^{b}$ Institute of Physics, Academia Sinica, \\ Taipei, Taiwan 11529, R.O.C. \\ ${ }^{c}$ Physics Division, National Center for Theoretical Sciences, \\ Hsinchu, Taiwan 30013, R.O.C. \\ ${ }^{d}$ Department of Physics, National Cheng Kung University, \\ 1, Ta-Hsueh Road, Tainan, Taiwan 70101, R.O.C. \\ E-mail: chengwei@ncu.edu.tw, nomura@ncu.edu.tw, keiyagyu@ncu.edu.tw
}

ABSTRACT: We study collider phenomenology of a leptophobic $Z^{\prime}$ boson existing in eight scenarios of the $E_{6}$ grand unified theory, differing in particle embeddings. We first review the current bound on the $Z^{\prime}$ mass $m_{Z^{\prime}}$ based upon the LHC data of $p p \rightarrow t \bar{t}$ process at $8 \mathrm{TeV}$ collisions with an integrated luminosity of $19.6 \mathrm{fb}^{-1}$. Most scenarios have a lower bound of about $1 \mathrm{TeV}$. However, this constraint does not apply to the case where $m_{Z^{\prime}}<2 m_{t}$, and other methods need to be employed for this lower mass regime. Using existing UA2 constraints and dijet data at the LHC, we find that only one of the eight scenarios is excluded at $95 \%$ confidence level. No bound can be obtained from $W j j$ and $Z j j$ measurements. We propose to use the photon associated production of the $Z^{\prime}$ boson that subsequently decays into a pair of bottom quarks, $p p \rightarrow Z^{\prime} \gamma \rightarrow b \bar{b} \gamma$, at the LHC to explore the constraints in the lower mass regime. We compute the expected signal significance as a function of $m_{Z^{\prime}}$ using detailed simulations of signal and irreducible background events. We find constraints for two more scenarios using the $8-\mathrm{TeV}$ data and taking appropriate kinematical cuts. We also show the discovery reach for each scenario at the $14-\mathrm{TeV}$ LHC machine.

KEywords: Phenomenological Models, Hadronic Colliders

ARXiv EPRINT: 1402.5579 


\section{Contents}

1 introduction 1

2 Leptophobic $Z^{\prime}$ boson 3

3 Constraints on the $Z^{\prime}$ mass by current data 5

3.1 The $p p \rightarrow t \bar{t}$ process 5

$\begin{array}{lll}3.2 & \text { The dijet process } & 7\end{array}$

3.3 The $W / Z$ plus dijet events 8

3.4 The constraints from UA2 9

$\begin{array}{ll}3.5 \text { Summary of constraints on } m_{Z^{\prime}} & 10\end{array}$

4 Photon associated production of $Z^{\prime} \quad 10$

$\begin{array}{lll}5 & \text { Conclusions } & 13\end{array}$

$\begin{array}{ll}\text { A Review of } E_{6} \text { GUT model } & 14\end{array}$

\section{1 introduction}

The operation of the 7- and 8-TeV runs of the CERN Large Hadron Collider (LHC) has provided us with quite important information about electroweak symmetry breaking; namely, the discovery of a standard model (SM)-like Higgs boson [1, 2] with a mass of about $126 \mathrm{GeV}$. This fact becomes a strong guidance for us to consider various models beyond the SM. Moreover, null results of any other new particles so far impose lower bounds on their masses and/or new physics scales. It is of great interest to discuss what kind of signals from new physics can be expected at the upcoming 13-and 14-TeV runs, while taking into account the data collected in the $8-\mathrm{TeV}$ run.

An extra $\mathrm{U}(1)$ gauge symmetry is often introduced based on various motivations in physics beyond the SM, resulting in an additional massive neutral gauge boson usually called the $Z^{\prime}$ boson. For example, there are usually additional $\mathrm{U}(1)$ gauge symmetries in grand unified theories (GUT's) such as the $E_{6}$ model [3-7]. Besides, the discrete $Z_{2}$ symmetry required for stabilizing dark matter candidates can naturally emerge from a local U(1) gauge group [8-15]. An extra U(1) has also been employed in supersymmetric models [1620] (so-called UMSSM) to facilitate a strong first order electroweak phase transition, as required to realize successful electroweak baryogenesis [21]. Properties of such $Z^{\prime}$ bosons strongly depend on the origin of the corresponding U(1) symmetry in models. Therefore, phenomenological studies of $Z^{\prime}$ bosons are essential to distinguish such new physics models (for nice and comprehensive reviews on $Z^{\prime}$ phenomenology in various models, please see, for example, [22, 23]). 
Searches for $Z^{\prime}$ bosons have been performed mainly using the dilepton events at the LHC. If the couplings of the $Z^{\prime}$ boson with fermions are the same as those of the $Z$ boson (the so-called sequential $Z^{\prime}$ case), the lower mass limit has been found to be $2.86 \mathrm{TeV}$ $(1.90 \mathrm{TeV})$ at the $95 \%$ confidence level (CL) from collisions at $8 \mathrm{TeV}$ with an integrated luminosity of $19.5 \mathrm{fb}^{-1}$ by using $e^{+} e^{-}$and $\mu^{+} \mu^{-}[24]\left(\tau^{+} \tau^{-}[25]\right)$ events.

However, such searches become ineffective when the $Z^{\prime}$ boson does not couple to the leptons. In this paper, we focus on the study of leptophobic $Z^{\prime}$ bosons derived from different scenarios of the $E_{6}$ GUT.

In the $E_{6}$ model $[26,27]$, there is a kinetic mixing between the hypercharge $\mathrm{U}(1)_{Y}$ group and the extra U(1)'s after GUT breaking. As a result, the $Z^{\prime}$ charge of each fermion is a linear combination of these $\mathrm{U}(1)$ charges, involving two free parameters. They can be chosen so that the $Z^{\prime}$ charges for the left-handed and right-handed charged leptons are zero, rendering the leptophobia nature.

Phenomenological studies of the leptophobic $Z^{\prime}$ boson had been done in refs. [28-40], with collider signals for the $Z^{\prime}$ searched for at the Tevatron [28-31] and the LHC [29-31]. LHC collider signatures of a leptophobic $Z^{\prime}$ boson that couples to a dark matter candidate had been studied in refs. [32, 33]. In refs. [34-36], a leptophobic $Z^{\prime}$ boson with the mass of about $150 \mathrm{GeV}$ was proposed to explain the excess in the $W j j$ events observed by the Tevatron CDF Collaboration. Effects of the leptophobic $Z^{\prime}$ to the $e^{+} e^{-} \rightarrow q \bar{q}$ process due to the $Z-Z^{\prime}$ mixing had been analyzed in ref. [37-40].

In this paper, we discuss all possible scenarios with a leptophobic $Z^{\prime}$ boson in the $E_{6}$ GUT model, differing in particle embeddings [41]. First, we consider the bound on the $Z^{\prime}$ mass according to current data of collider experiments. In most scenarios, the $Z^{\prime}$ can be excluded up to about $\mathcal{O}(1) \mathrm{TeV}$ by the $p p \rightarrow Z^{\prime} \rightarrow t \bar{t}$ data at the LHC. However, this method does not apply when the $Z^{\prime}$ mass is below the threshold for decaying into a pair of top quarks. We also take into account dijet data at the LHC and at the UA2, deriving respectively a lower bound of about $500 \mathrm{GeV}$ and $250 \mathrm{GeV}$ on the $Z^{\prime}$ mass only in one of the scenarios. In addition, although the $W j j$ and $Z j j$ processes have been measured at the LHC, no bound can be obtained currently because of a small $Z^{\prime}$ contribution to the cross sections compared to the experimental error bar. Therefore, we propose a promising channel, the photon associated production of $Z^{\prime}$, at the LHC to search for the leptophobic $Z^{\prime}$ boson with a mass smaller than $2 m_{t}$.

We further focus on the bottom quark pair decay mode of $Z^{\prime}, p p \rightarrow Z^{\prime} \gamma \rightarrow b \bar{b} \gamma$, for the advantage of using double b-tagging to reduce the background events. With a detailed simulation of signal and background events, we obtain the result of signal significance as a function of the $Z^{\prime}$ mass. In addition, we further estimate the integrated luminosity required for a 5 -sigma discovery for the 14 -TeV LHC.

The structure of this paper is organized as follows. We review the interaction Lagrangian for the leptophobic $Z^{\prime}$ boson in section 2, where the decay and production of the $Z^{\prime}$ are also discussed. The current bounds on the $Z^{\prime}$ mass from various experiments are reviewed in section 3. In section 4 , we propose to use the $p p \rightarrow Z^{\prime} \gamma \rightarrow b \bar{b} \gamma$ process to search for a light $Z^{\prime}$ boson. A detailed simulation is presented to show what constraints we could have using the current data and the prospect of detecting such a particle at the 
14-TeV LHC. Our findings are summarized in section 5. A brief review of the different leptophobic scenarios in the $E_{6}$ model is given in the appendix.

\section{Leptophobic $Z^{\prime}$ boson}

The interactions of the leptophobic $Z^{\prime}$ boson with SM quarks are given by

$$
\mathcal{L}=\sum_{q=u, d} g_{Z^{\prime}} \bar{q} \gamma^{\mu}\left(v_{q}-\gamma_{5} a_{q}\right) q Z_{\mu}^{\prime}
$$

where $u$ and $d$ represent the up- and down-type quarks, respectively. For simplicity, we assume no or at least negligible flavor-changing couplings. The vector coupling coefficient $v_{q}$ and the axial-vector coupling coefficient $a_{q}$ are related to the $Z^{\prime}$ charge $\bar{Q}_{f}$ of the quark $q$ by

$$
v_{q}=\frac{\bar{Q}_{Q}}{2}\left(1+\frac{\bar{Q}_{q}}{\bar{Q}_{Q}}\right), \quad a_{q}=\frac{\bar{Q}_{Q}}{2}\left(1-\frac{\bar{Q}_{q}}{\bar{Q}_{Q}}\right), \quad(\text { for } q=u, d) .
$$

The appendix briefly reviews the scenarios in the $E_{6}$ GUT model that realize leptophobia for the $Z^{\prime}$ boson, along with the corresponding $Z^{\prime}$ charges. We note here that the value of the gauge coupling constant $g_{Z^{\prime}}$ at the $\mathrm{TeV}$ scale can be predicted according to renormalization group running from the GUT scale, which depends on the details of matter contents and unification scale. As in ref. [42], we adopt for definiteness $g_{Z^{\prime}}=\sqrt{5 / 3} g^{\prime}$, where $g^{\prime}$ is the hypercharge coupling, for phenomenological analyses. In our paper, the non-SM fermions such as $h$ listed in table IV in the appendix are assumed to be so heavy that their effects on the $Z^{\prime}$ phenomenology can be safely neglected.

The decay rate of $Z^{\prime}$ into a quark pair is

$$
\begin{aligned}
\Gamma\left(Z^{\prime} \rightarrow q \bar{q}\right) & =g_{Z^{\prime}}^{2} \frac{m_{Z^{\prime}}}{4 \pi}\left[v_{q}^{2}\left(1+2 x_{q}\right)+a_{q}^{2}\left(1-4 x_{q}\right)\right] \sqrt{1-4 x_{q}} \\
& =g_{Z^{\prime}}^{2} \frac{m_{Z^{\prime}}}{4 \pi} \frac{\bar{Q}_{Q}^{2}}{2}\left[1+\left(\frac{\bar{Q}_{q}}{\bar{Q}_{Q}}\right)^{2}-x_{q}\left(1-\frac{\bar{Q}_{q}}{\bar{Q}_{Q}}\right)^{2}\right] \sqrt{1-4 x_{q}}, \quad \text { with } x_{q}=\frac{m_{q}^{2}}{m_{Z^{\prime}}^{2}} .
\end{aligned}
$$

Apart from $x_{q}$ that depends on the $Z^{\prime}$ mass, the terms inside the square brackets involve just the two $Z^{\prime}$ charge ratios, $\bar{Q}_{u} / \bar{Q}_{Q}$ and $\bar{Q}_{d} / \bar{Q}_{Q}$. Among the eight scenarios given in table 4, Scenario-I and Scenario-IV have the same $Z^{\prime}$ charges, and Scenario-II have the same charge ratios as them, so that the decay branching fractions are the same in these three scenarios. However, the total width and the production cross section for $Z^{\prime}$ can be different between Scenario-I or Scenario-IV and Scenario-II as they are affected by the overall $\bar{Q}_{Q}$ factor.

In figure 1 , we show the contour plot of the total branching fraction for the $Z^{\prime}$ decaying into a pair of down-type quarks, $\sum_{q=d, s, b} \mathcal{B}\left(Z^{\prime} \rightarrow q \bar{q}\right)$, on the $\left|\bar{Q}_{u} / \bar{Q}_{Q}\right|$ and $\bar{Q}_{d} / \bar{Q}_{Q}$ plane, taking $m_{Z^{\prime}}=1 \mathrm{TeV}$ as an example. Here we note that in the calculations of total width and branching fractions, the $Z^{\prime}$ boson is assumed to decay into only SM quarks. The reason we use the absolute value of $\bar{Q}_{u} / \bar{Q}_{Q}$ as the horizontal axis is simply because $\bar{Q}_{u}$ is always negative among the scenarios. The predictions for the various leptophobic scenarios are 


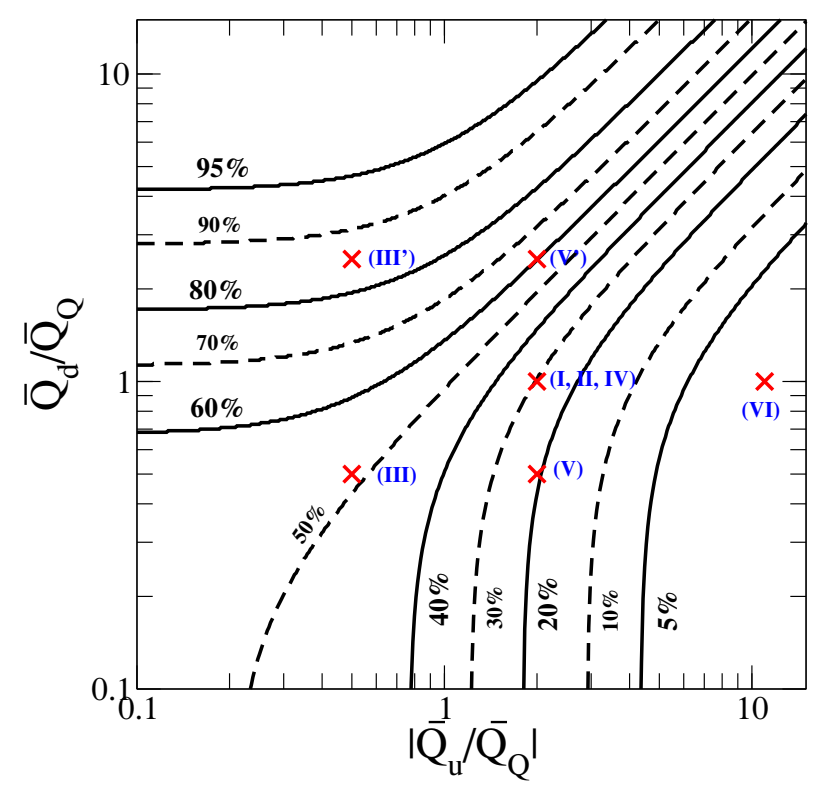

Figure 1. Contour plot of the total branching fraction for the $Z^{\prime}$ decaying into the down-type quarks on the $\left|\bar{Q}_{u} / \bar{Q}_{Q}\right|$ and $\bar{Q}_{d} / \bar{Q}_{Q}$ plane in the case of $m_{Z^{\prime}}=1 \mathrm{TeV}$. Predictions for the scenarios defined in table 4 in the appendix are indicated by the red crosses.

\begin{tabular}{|l|c|c|c|c|}
\hline Scenario & $u \bar{u}[\%]$ & $t \bar{t}[\%]$ & $d \bar{d}[\%]$ & width $[\mathrm{GeV}]$ \\
\hline Scenario-I & & & & $3.2,7.2,11.1$ \\
\cline { 1 - 3 } Scenario-II & $27.6,24.7,24.2$ & $11.8,20.8,22.5$ & $11.0,9.9,9.7$ & $0.32,0.72,1.1$ \\
\cline { 1 - 3 } & & & & $3.2,7.2,11.1$ \\
\hline Scenario-IV & & $7.9,14.4,15.7$ & $18.4,17.1,16.9$ & $0.73,1.6,2.4$ \\
\hline Scenario-III & $18.4,17.1,16.9$ & $2.2,4.2,4.6$ & $29.3,28.7,28.5$ & $2.7,5.4,8.2$ \\
\hline Scenario-III' & $5.0,4.9,4.9$ & 23.9 & $1.7,3.9,5.9$ \\
\hline Scenario-V & $31.5,27.8,27.2$ & $13.5,23.5,25.3$ & $7.9,7.0,6.8$ & $1.6,7.7,11.7$ \\
\hline Scenario-V' & $14.8,13.9,13.7$ & $6.3,11.7,12.8$ & $21.4,20.2,19.9$ & $3.6,7.7$ \\
\hline Scenario-VI & $37.9,34.0,33.3$ & $22.3,30.4,31.7$ & $0.6,0.6,0.5$ & $5.7,12.8,19.6$ \\
\hline
\end{tabular}

Table 1. Branching fractions and total width of $Z^{\prime}$ in the scenarios listed in table 4 . The column for $u \bar{u}(d \bar{d})$ displays the branching fractions for $Z^{\prime} \rightarrow u \bar{u}$ and $Z^{\prime} \rightarrow c \bar{c}\left(Z^{\prime} \rightarrow d \bar{d}, Z^{\prime} \rightarrow s \bar{s}\right.$ and $\left.Z^{\prime} \rightarrow b \bar{b}\right)$. The three numbers in each entry are predicted for $m_{Z^{\prime}}=500 \mathrm{GeV}, 1000 \mathrm{GeV}$ and $1500 \mathrm{GeV}$, respectively.

indicated by red crosses. The maximum (about 85\%) and the minimum (less than $5 \%$ ) are realized in Scenario-III' and Scenario-VI, respectively. We note that varying the $Z^{\prime}$ mass will cause shifts in the contours as a result of the $x_{q}$ dependence in eq. (2.3). The general tendency is that the total down-quark branching fraction becomes smaller for larger $Z^{\prime}$ mass, as reflected in table 1 , where the branching fraction of each mode and the total width are computed for $m_{Z^{\prime}}=500,1000$ and $1500 \mathrm{GeV}$.

In the following discussions, we will concentrate on the six scenarios that present distinct branching fraction patterns of the $Z^{\prime}$, i.e., Scenario-I, Scenario-III, Scenario-III', Scenario-V, Scenario-V', and Scenario-VI. 

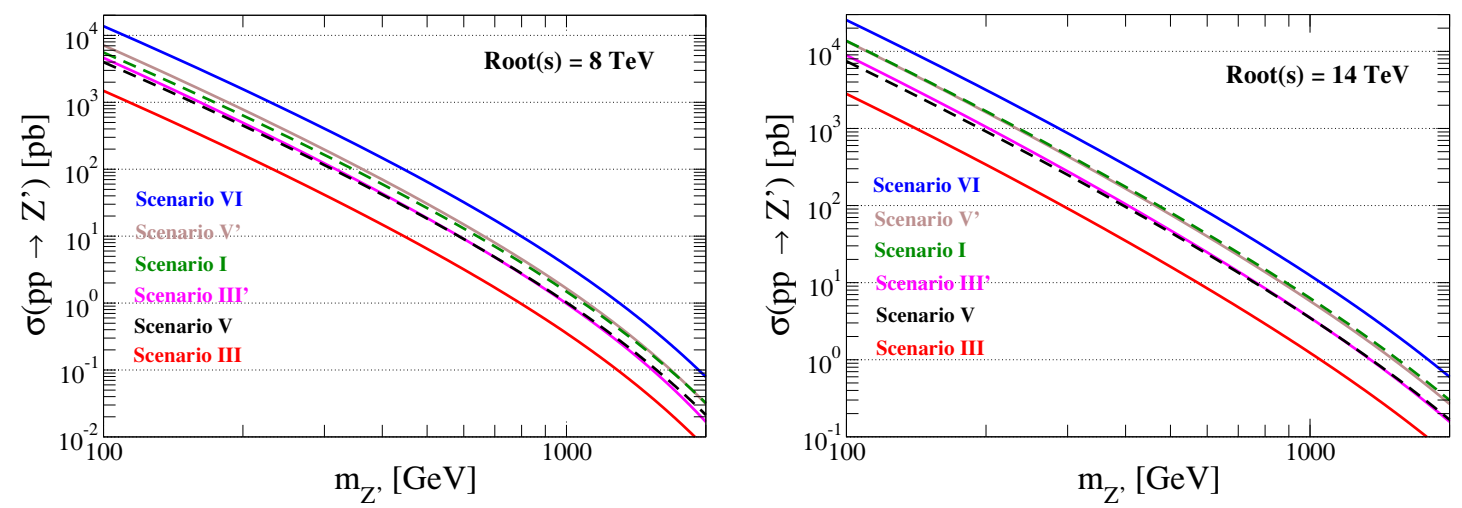

Figure 2. Production cross sections of the $p p \rightarrow Z^{\prime}$ process as a function of $m_{Z^{\prime}}$ for the collision energy of $8 \mathrm{TeV}$ (left) and $14 \mathrm{TeV}$ (right) in the six scenarios.

Dominant production mechanisms for the $Z^{\prime}$ at the LHC are the $s$-channel $p p \rightarrow Z^{\prime}$ process and the $t$-channel $p p \rightarrow Z^{\prime} V\left(V=\gamma, Z\right.$ and $\left.W^{ \pm}\right)$process of associated production. We calculate the production cross sections for these processes and those in the subsequent analyses with the help of CalcHEP [43] package and using CTEQ6L for the parton distribution functions (PDF's). In figure 2, the $s$-channel production cross section is shown as a function of $m_{Z^{\prime}}$ for the collision energy of $8 \mathrm{TeV}$ (left panel) and $14 \mathrm{TeV}$ (right panel). The biggest (smallest) cross section in the whole mass range is given by Scenario-VI (Scenario-III), because of the larger (smaller) $\bar{Q}_{u}$ charge for the up-type quarks.

In figure 3, the associated production cross sections for the $p p \rightarrow Z^{\prime} \gamma, p p \rightarrow Z^{\prime} Z$ and $p p \rightarrow Z^{\prime} W$ processes for each of the scenarios are shown as a function of $m_{Z^{\prime}}$ by the black, red and blue curves, respectively, also for the collision energy of $8 \mathrm{TeV}$ (dashed curves) and $14 \mathrm{TeV}$ (solid curves). For the $p p \rightarrow Z^{\prime} W$ process, the $W^{+}$and $W^{-}$contributions are summed over. We impose the $p_{T}(\gamma)>10 \mathrm{GeV}$ cut for the $p p \rightarrow Z^{\prime} \gamma$ process to avoid collinear singularity of the produced photon, where $p_{T}(\gamma)$ denotes the transverse momentum for the photon. In most cases, the cross sections are generally ranked in the order of $Z^{\prime} \gamma, Z^{\prime} W$ and $Z^{\prime} Z$ except for Scenario-VI. In the large $m_{Z^{\prime}}$ region, the produced vector boson tends to get smaller transverse mass, so that the production cross section of $Z^{\prime} \gamma$ reduces faster than those of $Z^{\prime} Z$ and $Z^{\prime} W$, as a result of the $p_{T}(\gamma)$ cut.

\section{Constraints on the $Z^{\prime}$ mass by current data}

In this section, we discuss various constraints on the $Z^{\prime}$ mass in the six scenarios. We consider the current data on $p p \rightarrow Z^{\prime} \rightarrow t \bar{t}$, dijet and $W / Z$ plus dijet processes at the LHC, as well as the $Z^{\prime} \bar{q} q$ couplings extracted from UA2 experiment.

\subsection{The $p p \rightarrow t \bar{t}$ process}

The CMS group reported the search for production of heavy resonances decaying into $t \bar{t}$ pairs in ref. [44]. They analyzed the events with one muon or electron and at least two jets in the final state using the data corresponding to an integrated luminosity of $19.6 \mathrm{fb}^{-1}$ at $8 \mathrm{TeV}$. Since no excess in events is observed, they provide an upper limit on the cross 

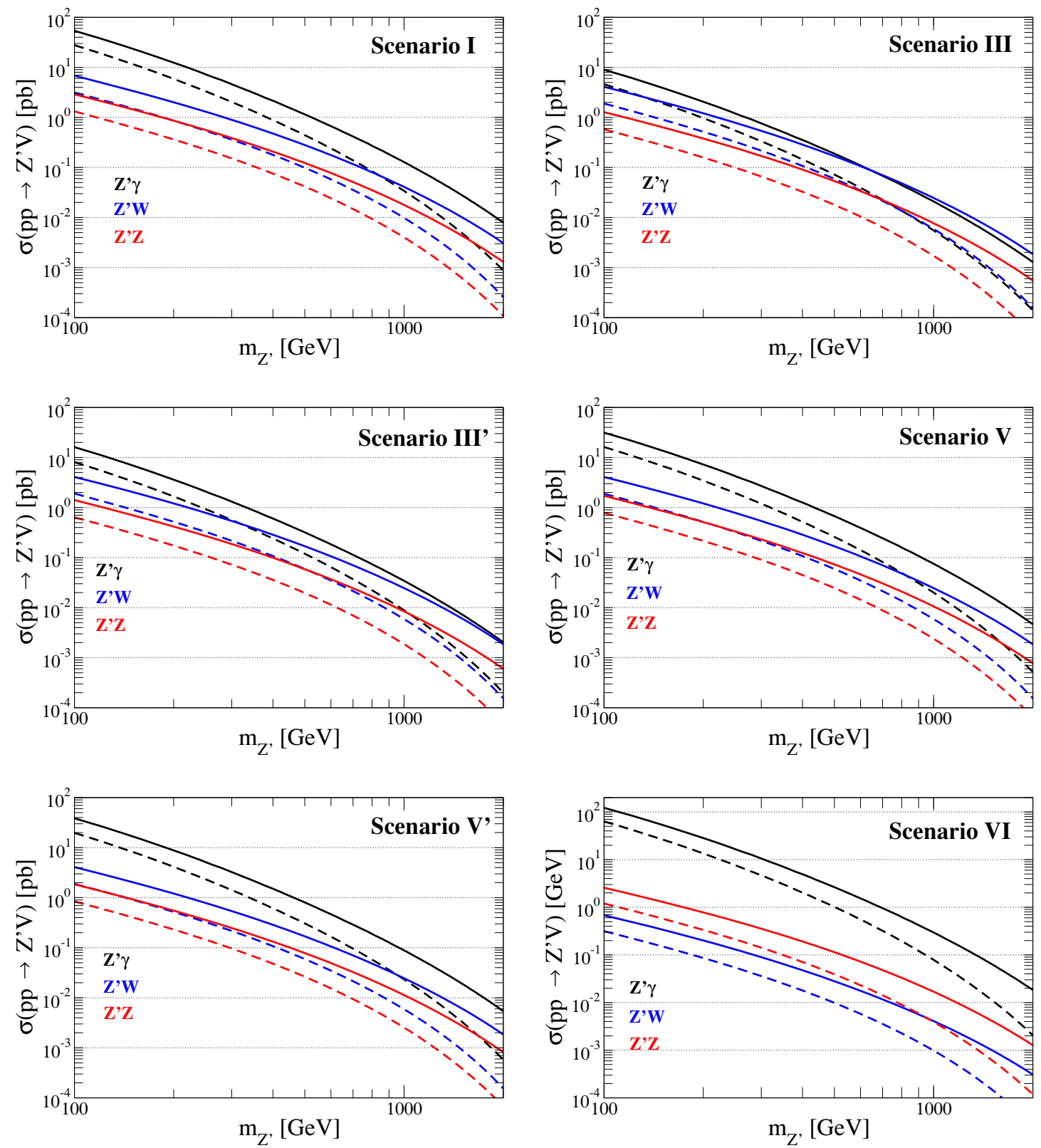

Figure 3. Cross sections of the $p p \rightarrow Z^{\prime} V\left(V=\gamma, Z\right.$ and $\left.W^{ \pm}\right)$process as a function of $m_{Z^{\prime}}$ for the collision energy of $8 \mathrm{TeV}$ (dashed curves) and $14 \mathrm{TeV}$ (solid curves). For the $p p \rightarrow Z^{\prime} \gamma$ process, we impose $p_{T}(\gamma)>10 \mathrm{GeV}$ to avoid the collinear singularity.

section of producing $t \bar{t}$ resonances at $95 \% \mathrm{CL}$ as a function of invariant mass of $t \bar{t}$ pair $M_{t \bar{t}}$. Comparing this limit with the cross section of $p p \rightarrow Z^{\prime} \rightarrow t \bar{t}$ in our scenarios, we can obtain a constraint on $m_{Z^{\prime}}$ by identifying $M_{t \bar{t}}$ as $m_{Z^{\prime}}$.

In figure 4 , we show the cross section of the $p p \rightarrow Z^{\prime} \rightarrow t \bar{t}$ process as a function of $m_{Z^{\prime}}$ in Scenarios-I, -III and -III' (left panel) and in Scenarios-V, -V' and -VI (right panel). The experimental upper limit for the cross section is indicated by the dashed curve. In this calculation, the narrow width assumption is employed; i.e., $\Gamma_{Z^{\prime}} / m_{Z^{\prime}}=1.2 \%$ with $\Gamma_{Z^{\prime}}$ 

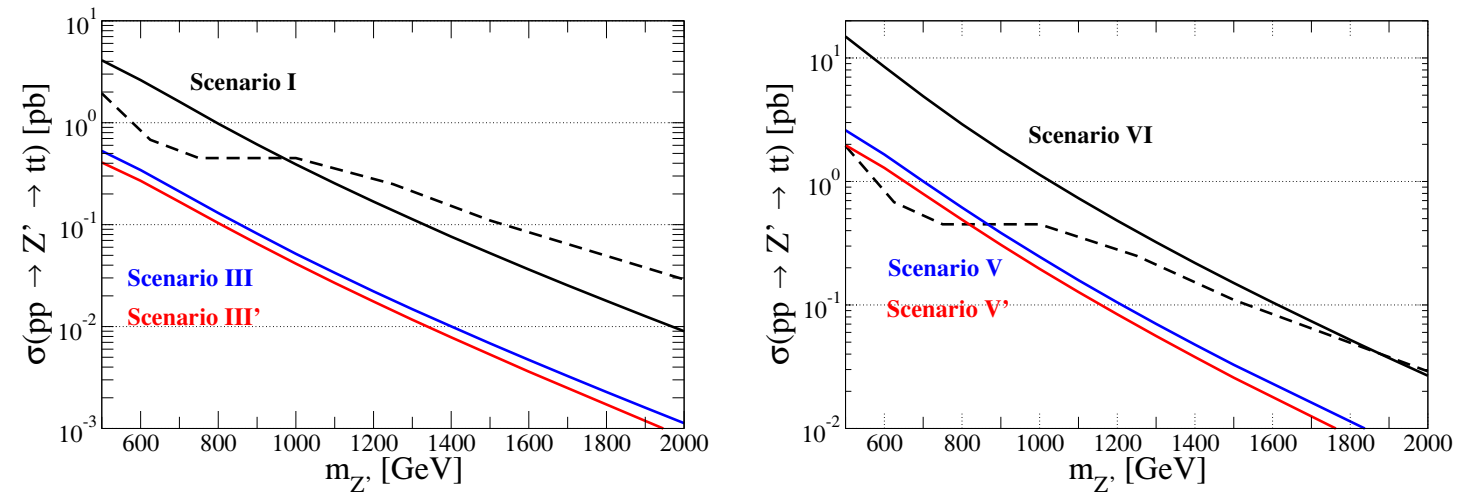

Figure 4. Cross section of the $p p \rightarrow Z^{\prime} \rightarrow t \bar{t}$ process as a function of $m_{Z^{\prime}}$ for the collision energy of $8 \mathrm{TeV}$. The left (right) panel shows the results in Scenario-I, -III and -III' (-V, -V' and -VI). The dashed curve is the observed limit at the $95 \%$ CL from the LHC data [44].

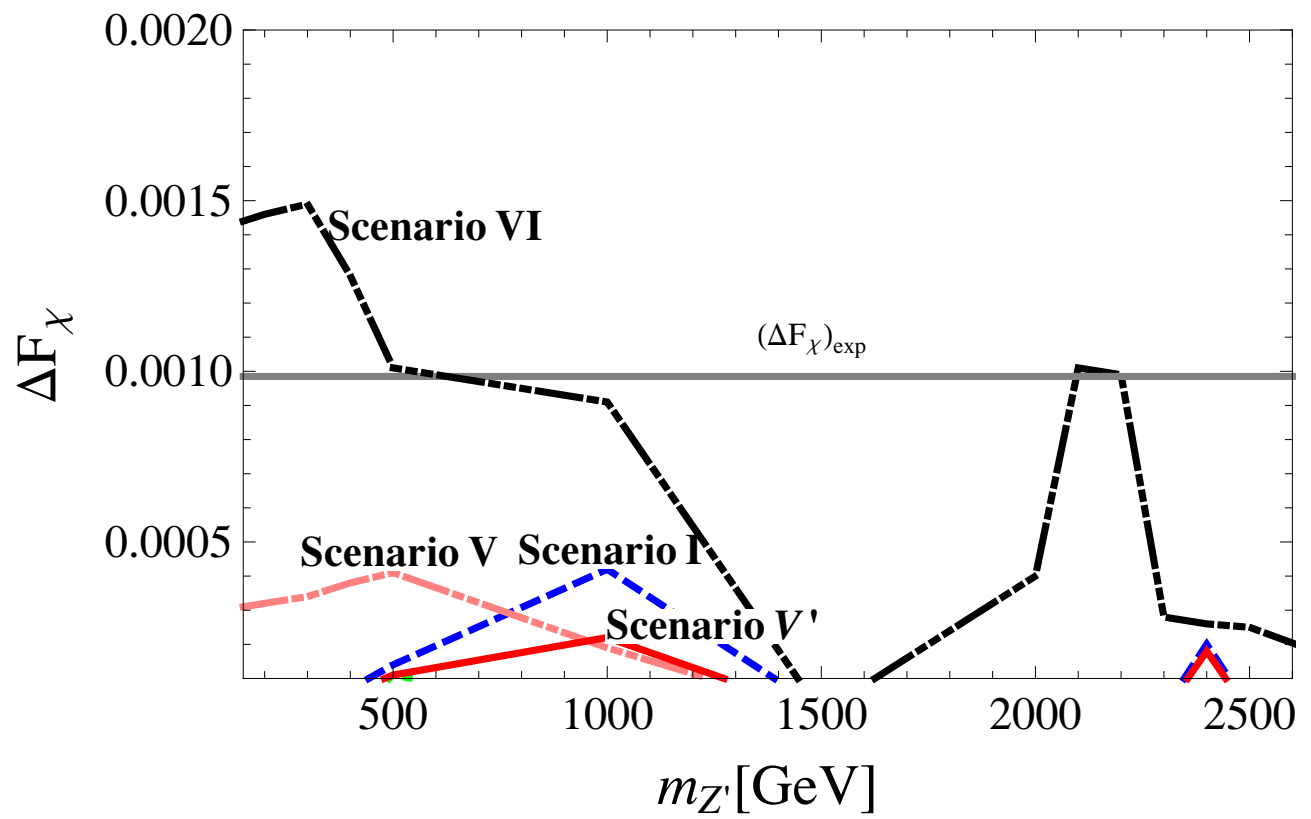

Figure 5. Values of $\Delta F_{\chi}$, defined in the text, in the different scenarios. The horizontal line corresponds to the upper limit for $\Delta F_{\chi}$ at $95 \%$ CL.

denoting the total width of $Z^{\prime}$, as used in ref. [44]. In Scenarios-I, -V, -V' and -IV, the lower bounds on $m_{Z^{\prime}}$ are extracted to be about $1000 \mathrm{GeV}, 850 \mathrm{GeV}, 800 \mathrm{GeV}$ and $1900 \mathrm{GeV}$, respectively. On the other hand, no constraint is obtained from this process in Scenario-III and Scenario-III'.

\subsection{The dijet process}

Dijet events at hadron colliders are useful for the detection and property analysis of a leptophobic $Z^{\prime}$ boson as one can readily measure a peak in the dijet invariant mass and study their angular distribution. Using the dijet resonant events at the LHC, ref. [45] put 
constraints on the mass and coupling constant for the leptophobic $Z^{\prime}$ boson. In ref. [46], it had been shown that the angular distribution of the dijet events with a high invariant mass could be used to probe the mass scales associated with different dimension- 6 operators by through effective Lagrangian approach. Particularly of interest to us is that the dijet process can receive contributions from four-quark interactions mediated by a $Z^{\prime}$ boson when the $Z^{\prime}$ mass is taken to be much larger than the typical jet momentum. Such a new interaction term can modify the dijet distribution from that of the QCD prediction. Thus, using the experimental dijet events, one can constrain the $Z^{\prime}$ mass provided the coupling constant is fixed.

The dijet measurement had been done at the LHC, offering various distributions [47, 48]. In order to compare the dijet events including the $Z^{\prime}$ mediation with the experimental data, we define $F_{\chi}$ value [47] as the ratio of two numbers of events in different regions of $\chi$ as

$$
F_{\chi}\left(m_{j j}^{\text {cut }}\right) \equiv \frac{N\left(\chi<3.32, m_{j j}^{\text {cut }}\right)}{N\left(\chi<30.0, m_{j j}^{\text {cut }}\right)},
$$

where $\chi \equiv \exp \left(\left|y_{1}-y_{2}\right|\right)$ with $y_{1,2}$ being the jet rapidities, and $m_{j j}^{\text {cut }}$ denotes an invariant mass cut for the dijet system. Ref. [48] provides the dijet event data with $m_{j j}^{\text {cut }}$ as $2 \mathrm{TeV}<$ $m_{j j}<2.6 \mathrm{TeV}$ based on the $4.8 \mathrm{fb}^{-1}$ data at $7 \mathrm{TeV}$. The central value of $F_{\chi}$ is extracted to be about 0.0848 .

We directly calculate the deviation in the value of $F_{\chi}$ from the SM prediction with the help of MADGRAPH [51] and CTEQ6L, instead of working with the effective Lagrangian. This is because the mass region considered here is not large enough compared to the dijet invariant mass. The deviation can be expressed as

$$
\Delta F_{\chi} \equiv \operatorname{MIN}\left|F_{\chi}^{Z^{\prime}}(1 \pm 0.02)-F_{\chi}^{\mathrm{SM}}(1 \pm 0.02)\right|
$$

where $F_{\chi}^{Z^{\prime}}$ and $F_{\chi}^{\mathrm{SM}}$ are respectively the values of $F_{\chi}\left(m_{j j}^{\text {cut }}\right)$ in the six scenarios of the leptophobic $Z^{\prime}$ and in the SM. In numerical calculations, there is about $2 \%$ uncertainty in the cross section. We therefore insert the factor $(1 \pm 0.02)$ and pick the minimum on the right-hand side. When the SM prediction is assumed to be the same as the experimental central value, one can set a $95 \%$ CL upper limit on $\Delta F_{\chi}$ by requiring $\Delta F_{\chi}<1.96 / \sqrt{N_{\exp }}$, where $N_{\exp }(=28462)$ is the number of events measured at the LHC [48].

In figure 5 , we show values of $\Delta F_{\chi}$ as a function of $m_{Z^{\prime}}$ for the six scenarios. The $95 \%$ CL upper limit for $\Delta F_{\chi}$ is indicated by the horizontal line. As shown in this figure, a $Z^{\prime}$ with mass smaller than about $500 \mathrm{GeV}$ is excluded only in Scenario-VI. On the other hand, no significant deviation in $F_{\chi}$ can be found in all the other scenarios. The peak at around $2 \mathrm{TeV}$ for Scenario-VI is due to our choice of the dijet invariant mass cut, $2 \mathrm{TeV} \leq m_{j j} \leq 2.6 \mathrm{TeV}$.

\subsection{The $W / Z$ plus dijet events}

The $W b \bar{b}$ and $Z b \bar{b}$ events had been measured at the LHC. The measured cross sections of $p p \rightarrow W b \bar{b} \rightarrow \mu \nu b \bar{b}$ and $p p \rightarrow Z b \bar{b} \rightarrow \ell^{+} \ell^{-} b \bar{b}(\ell$ is $e$ or $\mu$ ) processes are given as $0.53 \pm$ 0.05 (stat.) \pm 0.09 (syst.) \pm 0.06 (th.) \pm 0.05 (lum.) pb [49] and $0.37 \pm 0.01$ (stat.) \pm 0.07 (syst.) 


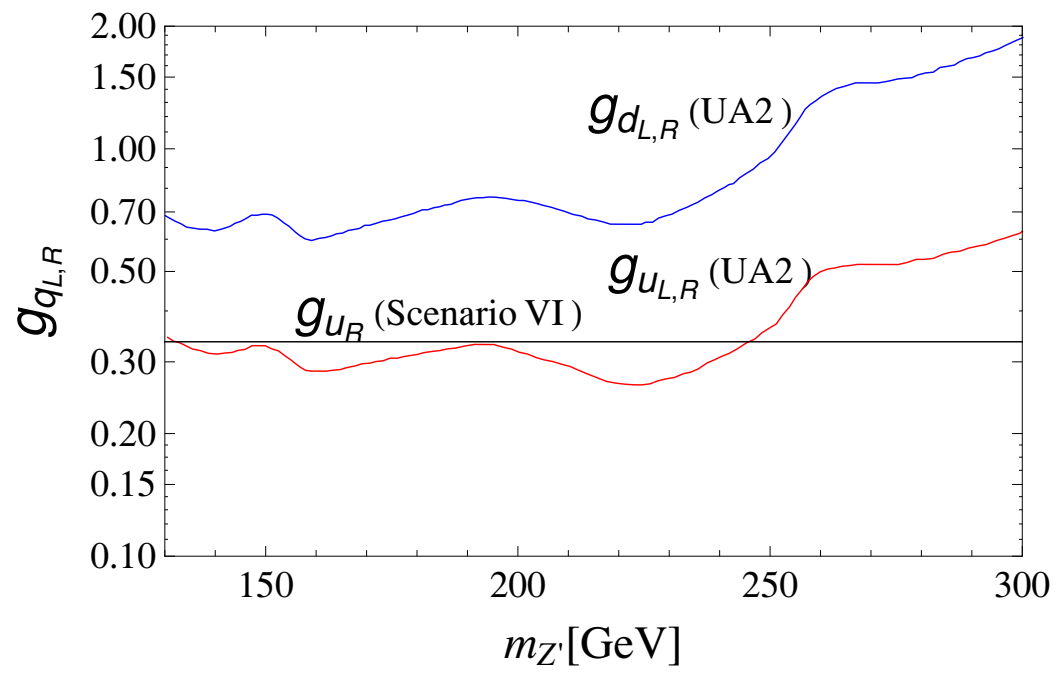

Figure 6. Constraints on the $Z^{\prime} \bar{q} q$ couplings (blue curve for the down-type quarks and red curve for the up-type quarks) taken from ref. [34] compared with $g_{u_{R}}$ in Scenario-VI (black horizontal line).

$\mathrm{pb}$ [50], respectively, with $7 \mathrm{TeV}$ and $5.0 \mathrm{fb}^{-1}$. According to ref. [49], the cross section of the $W b \bar{b}$ event has been obtained by taking the kinematical cuts $p_{T}>25 \mathrm{GeV}$ and $|\eta|<2.1$ for muon, and $p_{T}>25 \mathrm{GeV}$ and $|\eta|<2.4$ for b-tagged jets. On the other hand, the following cuts are imposed to obtain the cross section of the $Z b \bar{b}$ event: $p_{T}>20 \mathrm{GeV},|\eta|<2.4$ and $76<M_{\ell \ell}<106 \mathrm{GeV}$ for charged leptons, and $p_{T}>25 \mathrm{GeV}$ and $|\eta|<2.1$ for b-tagged jets $[50]$.

We calculate the cross sections of the $p p \rightarrow W b \bar{b} \rightarrow \mu \nu b \bar{b}$ and $p p \rightarrow Z b \bar{b} \rightarrow \ell^{+} \ell^{-} b \bar{b}$ processes in the SM and in Scenario-I with $m_{Z^{\prime}}=100 \mathrm{GeV}$ using MADGRAPH [51]. We find that the deviation in these cross sections are less than $0.01 \mathrm{pb}$ and $0.002 \mathrm{pb}$ for $W b \bar{b}$ and $Z b \bar{b}$ processes, respectively, which are much smaller than the above-mentioned experimental errors. Similar results can be obtained in all the other scenarios and heavier $Z^{\prime}$ masses. Thus, we cannot obtain any useful constraint on $m_{Z^{\prime}}$ using the current data of these processes.

The CMS group also analyzed $W j j$ events with the invariant mass of the dijet system $M_{j j}$ to be about $150 \mathrm{GeV}$. This process can receive contributions from the leptophobic $Z^{\prime}$; i.e., $p p \rightarrow W Z^{\prime} \rightarrow W j j$. A cross section of $8.1 \mathrm{pb}$ is expected in order to explain the CDF anomaly, but is excluded using the data sample of $5 \mathrm{fb}^{-1}$ at $7 \mathrm{TeV}$ [52]. In all our scenarios, the $W Z^{\prime}$ production cross section is smaller than $1 \mathrm{pb}$ as shown in figure 3. Consequently, the $Z^{\prime}$ boson in our scenarios is not constrained by current $W b \bar{b} / Z b \bar{b}$ and $W j j / Z j j$ events. As we will show in the next section, the $p p \rightarrow Z^{\prime} \gamma$ process serves more useful as it gives a larger cross section.

\subsection{The constraints from UA2}

The dijet invariant mass spectrum of $p \bar{p} \rightarrow 2$ jets had been measured in the UA2 experiment at CERN with the data sample of $10.9 \mathrm{pb}^{-1}$, in search of any extra heavy vector bosons 


\begin{tabular}{|lcccccc|}
\hline & Scenario-I & Scenario-III & Scenario-III' & Scenario-V & Scenario-V' & Scenario-VI \\
\hline$p p \rightarrow t \bar{t}$ & $1000 \mathrm{GeV}$ & - & - & $800 \mathrm{GeV}$ & $850 \mathrm{GeV}$ & $1900 \mathrm{GeV}$ \\
dijet & - & - & - & - & - & $500 \mathrm{GeV}$ \\
UA2 & - & - & - & - & - & $250 \mathrm{GeV}$ \\
\hline
\end{tabular}

Table 2. Lower bounds on $m_{Z^{\prime}}$ at $95 \%$ CL for all the scenarios by existing experiments. The bounds from $p p \rightarrow t \bar{t}$ are only valid for $m_{Z^{\prime}}>2 m_{t}$.

that decay into two jets [53]. The fact that no excess had been observed can be converted into a constraint on the $Z^{\prime}$ couplings with quarks in the mass range $130<m_{Z^{\prime}}<300 \mathrm{GeV}$. In ref. [34], constraints on the chiral couplings in

$$
g_{q_{L, R}} \bar{q} \gamma^{\mu} \frac{1 \mp \gamma_{5}}{2} q Z_{\mu}^{\prime}, \text { with } q=u, d,
$$

had been given in figure 1 by comparing the cross section for the $p \bar{p} \rightarrow Z^{\prime} \rightarrow j j$ process with that of the upper limit obtained from the UA2 experiment at the center-of-mass energy of $630 \mathrm{GeV}$. The chiral couplings expressed in eq. (3.3) are related to the variables in eq. (2.1) by

$$
g_{q_{L, R}}=g_{Z^{\prime}}\left(v_{q} \pm a_{q}\right)
$$

where the values of $v_{q}$ and $a_{q}$ are given by eq. (2.2) and table 4 in the appendix. In figure 6, we show the upper limits for $g_{u_{R, L}}$ and $g_{d_{R, L}}$ extracted from figure 1 of ref. [34], in comparison with $g_{u_{R}}$ in Scenario-VI indicated by the horizontal line. The coupling constant $g_{u_{L}}$ in Scenario-VI and that including all the other couplings in the other scenarios are well below these constraints and thus omitted. We therefore obtain the excluded region of $m_{Z^{\prime}} \lesssim 250 \mathrm{GeV}$ in Scenario-VI.

\subsection{Summary of constraints on $m_{Z^{\prime}}$}

Here we summarize the constraints on $m_{Z^{\prime}}$ discussed in this section. The lower bounds on $m_{Z^{\prime}}$ at $95 \%$ CL for all the scenarios are listed in table 2 . The constraint from the $p p \rightarrow t \bar{t}$ process is the strongest among all. However, this constraint is valid only when the $Z^{\prime} \rightarrow t \bar{t}$ decay is kinematically allowed. On the other hand, the constraints from the dijet events and the UA2 constraint can be applied to the lighter $Z^{\prime}$ case; namely, $m_{Z^{\prime}}<2 m_{t}$. Except for Scenario-VI, all other scenarios are not restricted by them mainly because of the small $Z^{\prime}$ charges for up-type quarks. We also find that it is currently difficult to extract constraints on the $Z^{\prime}$ mass from the $Z b \bar{b}, W b \bar{b}, Z j j$ and $W j j$ events because of the small cross sections of $Z Z^{\prime}$ and $W Z^{\prime}$ production. In the next section, we study the light $Z^{\prime}$ case using the $t$-channel $p p \rightarrow Z^{\prime} \gamma$ process.

\section{Photon associated production of $Z^{\prime}$}

In this section, we propose to use the $t$-channel process $p p \rightarrow Z^{\prime} \gamma \rightarrow b \bar{b} \gamma$ to search for a relatively light $Z^{\prime}$ with $m_{Z^{\prime}} \lesssim 350 \mathrm{GeV}$, where the $Z^{\prime} \rightarrow t \bar{t}$ decay is kinematically forbidden. Since no experimental data exist at the time of writing, we present a simulation study here. 


\begin{tabular}{|lcccc|}
\hline & $\gamma Z^{\prime}(\rightarrow b \bar{b})$ & $\gamma b \bar{b}$ & $\gamma j j$ & $\mathcal{S}$ \\
\hline Basic cuts in eq. (4.1) & 0.256 & 21.8 & 2952 & 0.66 \\
Double b-tagging & 0.0269 & 2.39 & 3.60 & 1.54 \\
$M_{b \bar{b}}$ cut in eq. (4.2) & 0.015 & 0.449 & 0.541 & 2.14 \\
\hline
\end{tabular}

Table 3. Cross sections of signal and background processes in Scenario-I in units of pb, assuming $m_{Z^{\prime}}=200 \mathrm{GeV}$ and $\sqrt{s}=8 \mathrm{TeV}$ as an example. To calculate the significance $\mathcal{S}$, we take an integrated luminosity of $19.6 \mathrm{fb}^{-1}$. The basic cuts eq. (4.1) are imposed, and the double b-tagging is applied after PGS detector simulations.

With b-tagging, the $Z^{\prime} \rightarrow b \bar{b}$ decay is expected to have higher sensitivity than the $Z^{\prime} \rightarrow q \bar{q}$ decays, where $q$ refers to quarks in the first and second generations. For the backgrounds, we include the SM irreducible background $p p \rightarrow b \bar{b} \gamma$ and the $p p \rightarrow q \bar{q} \gamma$ process with mis-tagging of the $b$ quarks.

In our analysis, we generate signal and background events using MADGRAPH/MADEVENT [51] and the CTEQ6L PDF's for the collision energies $\sqrt{s}=8 \mathrm{TeV}$ and $14 \mathrm{TeV}$. The generated events are passed onto PYTHIA [54] through the PYTHIA-PGS package to include initial-state radiation, final-state radiation and hadronization effects. The detector level simulation is then carried out by PGS [55]. For the generated events, we first apply the following basic kinematical cuts:

$$
\begin{array}{rlrl}
p_{T}(\text { jets }) & >40 \mathrm{GeV}, & & p_{T}(\gamma)>10 \mathrm{GeV}, \\
\mid \eta(\text { jets }) \mid & <2.4, & & \left|\Delta \eta_{j j}\right|<2.4, \\
90 \mathrm{GeV} & <M_{j j}<360 \mathrm{GeV}, &
\end{array}
$$

where the jets include b-jets, and $\left|\Delta \eta_{j j}\right|$ is the rapidity difference of the two jets. The $M_{j j}$ cut restricts ourselves to the mass regime $100 \mathrm{GeV} \lesssim m_{Z^{\prime}} \lesssim 360 \mathrm{GeV}$. Moreover, we impose double b-tagging after the PGS detector simulation to reduce the background events. Afterwards, we further take a cut on the invariant mass of the two b-jets $M_{b \bar{b}}$ :

$$
m_{Z^{\prime}}(1-0.2)<M_{b \bar{b}}<m_{Z^{\prime}}+10 \mathrm{GeV}
$$

for each $m_{Z^{\prime}}$ value. ${ }^{1}$ We then calculate the signal significance defined by [56]

$$
\mathcal{S}=\sqrt{2[(s+b) \ln (1+s / b)-s]}
$$

where $s$ and $b$ are the numbers of signal and background events, respectively. In table. 3, we show as an example how the cuts affect the number of events in Scenario-I, assuming $m_{Z^{\prime}}=200 \mathrm{GeV}$ and $\sqrt{s}=8 \mathrm{TeV}$. The $\gamma j j$ background is significantly reduced by about three orders of magnitude by double b-tagging while the signal and irreducible background are down by one order of magnitude, thereby enhancing the significance by more than a

\footnotetext{
${ }^{1}$ Because the shape of the $b \bar{b}$ invariant mass distribution is asymmetric around $M_{b \bar{b}}=m_{Z^{\prime}}$, we use different upper and lower cut limits in eq. (4.2). The asymmetric distribution is due to the fact that signal events tend to shift to lower $M_{b b}$ at the detector level, mainly as a result of soft radiation from the b-jets.
} 

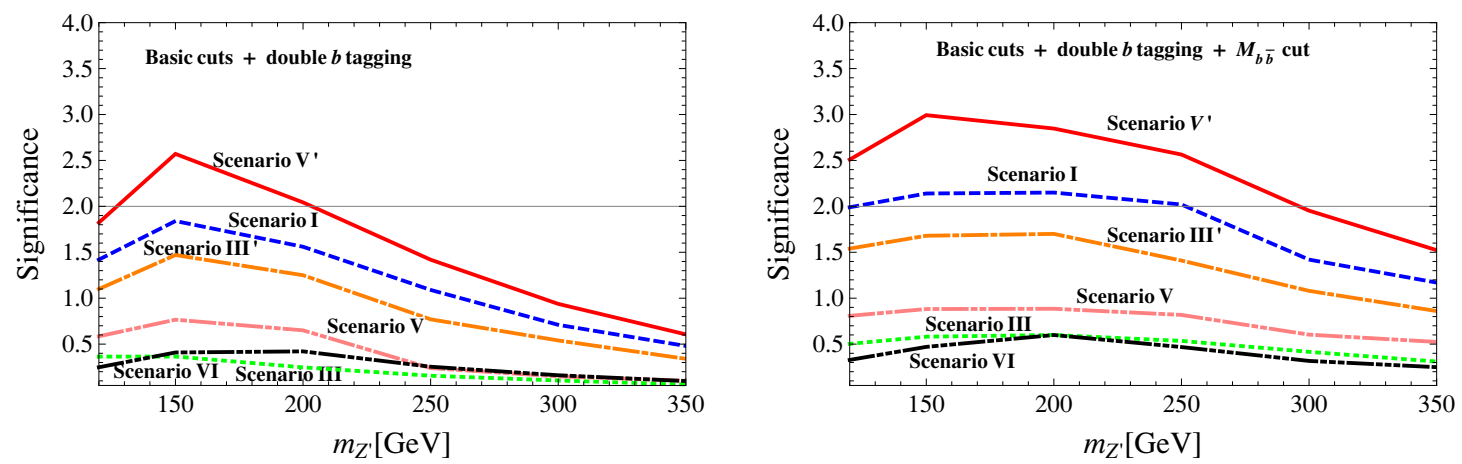

Figure 7. Significance for the $p p \rightarrow Z^{\prime} \gamma \rightarrow b \bar{b} \gamma$ process at the LHC with $\sqrt{s}=8 \mathrm{TeV}$ and an integrated luminosity of $19.6 \mathrm{fb}^{-1}$. The left panel shows the result after imposing the basic cuts and double b-tagging, and the right panel that after taking the $M_{b \bar{b}}$ cut.
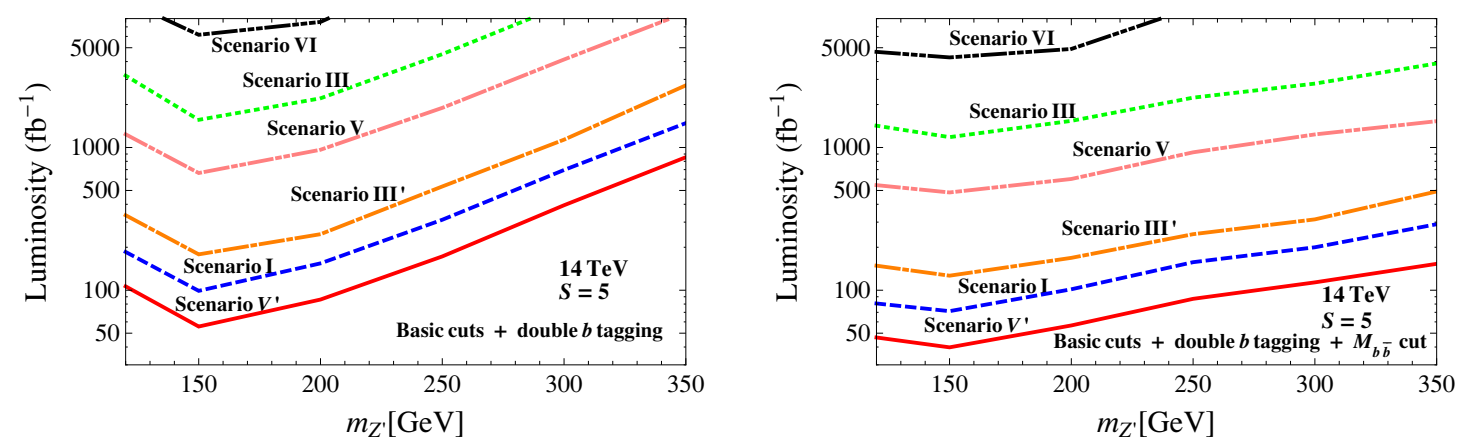

Figure 8. Required integrated luminosity for 5-sigma discovery $(\mathcal{S}=5)$ from the $p p \rightarrow Z^{\prime} \gamma \rightarrow b \bar{b} \gamma$ process at the LHC with $\sqrt{s}=14 \mathrm{TeV}$. The left panel shows the result after imposing the basic cuts and double b-tagging, and the right panel that after taking the $M_{b \bar{b}}$ cut.

factor of two. We also observe that the $M_{b \bar{b}}$ cut is effective in reducing not only the $\gamma j j$ background but also the $b \bar{b} \gamma$ background.

In figure 7 , we show the signal significance for the six scenarios as a function of $m_{Z^{\prime}}$, assuming $\sqrt{s}=8 \mathrm{TeV}$ and an integrated luminosity of $19.6 \mathrm{fb}^{-1}$. The left panel shows the result after imposing the basic cuts and double b-tagging, and the right panel that after taking the $M_{b \bar{b}}$ cut. Assuming the data are consistent with SM prediction, we find that the mass range of $130 \mathrm{GeV} \lesssim m_{Z^{\prime}} \lesssim 200 \mathrm{GeV}$ can be excluded at 95\% CL for Scenario-V' using the basic cuts and double b-tagging. After further imposing the $M_{b \bar{b}}$ cut, the mass range of $120 \mathrm{GeV} \lesssim m_{Z^{\prime}} \lesssim 290(240) \mathrm{GeV}$ can be excluded for Scenario-V' (Scenario-I) at 95\% CL. The other scenarios will be less constrained by this analysis. The hierarchy in the significances of the different scenarios depends on the product of the cross section of $p p \rightarrow Z^{\prime} \gamma$, shown in figure 3 , and the branching fraction of $Z^{\prime} \rightarrow b \bar{b}$, given in table. 1 and figure 1.

To study the discovery reach for the $14-\mathrm{TeV}$ LHC, we compute the required integrated luminosity to reach $S=5$ as a function of $m_{Z^{\prime}}$. The left panel in figure 8 shows the result after imposing the basic cuts and double b-tagging. The right panel shows the result after further taking the $M_{b \bar{b}}$ cut. We thus find that a 5 -sigma discovery can be obtained for 
$m_{Z^{\prime}} \lesssim 200 \mathrm{GeV}$ in Scenario-V' with an integrated luminosity of $100 \mathrm{fb}^{-1}$ by applying only the basic cuts and double b-tagging. With further the $M_{b \bar{b}}$ cut, the reach can be extended to $m_{Z^{\prime}} \lesssim 290 \mathrm{GeV}$ in Scenario-V' and $m_{Z^{\prime}} \lesssim 190 \mathrm{GeV}$ in Scenario-I. With an integrated luminosity of $500 \mathrm{fb}^{-1}$ and all cuts mentioned above, we can cover the entire mass range in the plots for Scenarios-V', -I, and -III'.

Finally, we would like to comment on the search for the leptophobic $Z^{\prime}$ boson at the international linear collider (ILC). ${ }^{2}$ Even though the ILC is an electron-positron collider where the initial leptons do not couple to the leptophobic $Z^{\prime}$ boson, it can still be produced in association with the quark pair production, i.e., $e^{+} e^{-} \rightarrow \gamma^{*} / Z^{*} \rightarrow q \bar{q} Z^{\prime}$. There are several designs of the ILC collision energy, namely, $250 \mathrm{GeV}, 350 \mathrm{GeV}$ and $500 \mathrm{GeV}$. Therefore, the $Z^{\prime}$ boson with a mass smaller than these energies can be produced in association with the light quark pair. ${ }^{3}$ In particular, it would be interesting to analyze the case with $m_{Z^{\prime}}<2 m_{t}$ because it is difficult to probe using the current experimental data, as we have discussed in the previous section. This will lead to the signal of four hard jets with the invariant mass of two sitting at the $Z^{\prime}$ mass. For example, the cross section of $e^{+} e^{-} \rightarrow q \bar{q} Z^{\prime}$ with $m_{Z^{\prime}}=150 \mathrm{GeV}$ at a $250-\mathrm{GeV}$ ILC is estimated to be $0.1 \mathrm{fb}$. If such a $Z^{\prime}$ is discovered in the $14-\mathrm{TeV}$ LHC, one may use the above-mentioned process to study detailed properties of the new boson. Even in the case where such a boson is not found at the LHC and the exclusion limit has not reached the $95 \% \mathrm{CL}$, one can use the this process as a discovery channel. In any case, it is important to prepare the simulation study for the $Z^{\prime}$ boson at the ILC. A detailed analysis for leptophobic $Z^{\prime}$ searches at the ILC will be presented in a separate work [58].

\section{Conclusions}

We have studied the phenomenology of a leptophobic $Z^{\prime}$ boson that can be derived from the $E_{6}$ GUT model, as a result of kinetic mixing between $\mathrm{U}(1)_{Y}$ and the extra U(1) symmetries. Due to different embedding schemes for the matter fields in the $E_{6}$ fundamental representation, there are eight possible scenarios with a leptophobic $Z^{\prime}$, differing in the $Z^{\prime}$ charges for the quarks and the exotic fermion. Three of the scenarios have the same $Z^{\prime}$ charge ratios. Therefore, the production cross sections of these scenarios can be related to one another by simple scaling. This reduces the number of distinct scenarios to six.

We have taken into account current experimental data to constrain the $Z^{\prime}$ mass, including $p p \rightarrow t \bar{t}$, the dijet events, $W / Z$ plus dijet events and the UA2 data. When the top pair decay of $Z^{\prime}$ is kinematically allowed, the strongest bound on the $Z^{\prime}$ mass comes from the $p p \rightarrow t \bar{t}$ process. In this case, the lower bounds on $m_{Z^{\prime}}$ at $95 \% \mathrm{CL}$ are about $1 \mathrm{TeV}$, 0.8 TeV, $0.85 \mathrm{TeV}$ and $1.9 \mathrm{TeV}$ in Scenario-I, Scenario-V', Scenario-V and Scenario-VI, respectively. However, this channel is not effective when $m_{Z^{\prime}}<2 m_{t}$. On the other hand, only Scenario-VI is constrained by the dijet and UA2 data, from which the lower limits are given as $500 \mathrm{GeV}$ and $250 \mathrm{GeV}$, respectively. We also found that it is difficult to obtain constraints on $Z^{\prime}$ mass from $W / Z$ plus dijet events due to the small cross sections of $Z Z^{\prime}$ and $W Z^{\prime}$ production.

\footnotetext{
${ }^{2}$ Recently, phenomenology of $Z^{\prime}$ at the ILC has been discussed in ref. [57] in a model-independent way using the $e^{+} e^{-} \rightarrow \mu^{+} \mu^{-} / \tau^{+} \tau^{-}$processes.

${ }^{3}$ Although one can consider the $e^{+} e^{-} \rightarrow t \bar{t} Z^{\prime}$ process, it suffers from a kinematical disadvantage.
} 
We have proposed to use the photon associated production of the $Z^{\prime}$ boson followed by the decay into a pair of bottom quarks, i.e., $p p \rightarrow \gamma Z^{\prime} \rightarrow \gamma b \bar{b}$ to explore the constraints in the lower mass regime, particularly for scenarios other than Scenario-VI. Specifying the decay of $Z^{\prime}$ into a pair of $b$ quarks helps reducing background events significantly. We have performed a detailed simulation of signal and irreducible background events, and searched for appropriate kinematical cuts to increase signal significance. We have found that Scenario-I (usually called the standard $E_{6}$ ) with $m_{Z^{\prime}} \lesssim 250 \mathrm{GeV}$ can be excluded at $95 \% \mathrm{CL}$ after imposing all the cuts on the current $\mathrm{LHC} 19.6 \mathrm{fb}^{-1}$ data at $8 \mathrm{TeV}$. A similar bound of $m_{Z^{\prime}} \lesssim 300 \mathrm{GeV}$ has also been obtained for Scenario-V'. Assuming an integrated luminosity of $100 \mathrm{fb}^{-1}$ for the 14 -TeV LHC, a 5-sigma discovery can be reached for $m_{Z^{\prime}} \lesssim 290 \mathrm{GeV}$ in Scenario-V' and $m_{Z^{\prime}} \lesssim 190 \mathrm{GeV}$ in Scenario-I.

\section{Acknowledgments}

C.-W. C would like to thank D. Choudhury and N. Gaur for useful discussions during the very early stage of this project. This research was supported in part by the National Science Council of R. O. C. under Grant Nos. NSC-100-2628-M-008-003-MY4, NSC-1012811-M-008-014 and NSC-102-2811-M-006-035.

\section{A Review of $E_{6}$ GUT model}

We present a brief review of scenarios in the $E_{6}$ GUT model that predict a leptophobic $Z^{\prime}$ boson, with its detailed derivations and fermion interactions given in ref. [42]. The symmetry breaking of $E_{6}$ follows the pattern of

$$
E_{6} \rightarrow \mathrm{SO}(10) \times \mathrm{U}(1)_{\psi} \rightarrow \mathrm{SU}(5) \times \mathrm{U}(1)_{\chi} \times \mathrm{U}(1)_{\psi} \rightarrow G_{\mathrm{SM}} \times \mathrm{U}(1)_{Q^{\prime}}
$$

The $\mathrm{U}(1)_{Q^{\prime}}$ symmetry is obtained as a linear combination of $\mathrm{U}(1)_{\psi}$ and $\mathrm{U}(1)_{\chi}$, with the corresponding charge expressed as

$$
Q^{\prime}=Q_{\psi} \cos \theta-Q_{\chi} \sin \theta
$$

where $Q_{\psi}$ and $Q_{\chi}$ are the charges under $\mathrm{U}(1)_{\psi}$ and $\mathrm{U}(1)_{\chi}$, respectively.

The most general kinetic terms, including kinetic mixing, for the gauge fields and interaction terms for a fermion $\psi$ are given by

$$
\begin{aligned}
\mathcal{L}_{\text {kin }} & =-\frac{1}{4} W_{\mu \nu}^{a} W^{a \mu \nu}-\frac{1}{4}\left(\tilde{B}_{\mu \nu}, \tilde{Z}_{\mu \nu}^{\prime}\right)\left(\begin{array}{cc}
1 & \sin \chi \\
\sin \chi & 1
\end{array}\right)\left(\begin{array}{c}
\tilde{B}^{\mu \nu} \\
\tilde{Z}^{\prime} \mu \nu
\end{array}\right), \\
\mathcal{L}_{\text {int }} & =-\bar{\psi} \gamma^{\mu}\left(g T^{a} W_{\mu}^{a}+g^{\prime} Y \tilde{B}_{\mu}+\tilde{g}_{Z^{\prime}} Q^{\prime} \tilde{Z}^{\prime}{ }_{\mu}\right) \psi
\end{aligned}
$$

where $g$ and $g^{\prime}$ are the $\mathrm{SM} \mathrm{SU}(2)_{L}$ and $\mathrm{U}(1)_{Y}$ gauge couplings, and $Y$ is the $\mathrm{SM} \mathrm{U}(1)_{Y}$ hypercharge. ${ }^{4}$ We note in passing that the kinetic mixing can be obtained at oneloop level with the matter contents of non-zero $Q^{\prime}$ and $Y$ charges running inside the loop $[59,60]$. In this case, the kinetic mixing $\sin \chi$ would be proportional to the factor

\footnotetext{
${ }^{4}$ We do not pull out the factors of $\sqrt{3 / 5}$ and $\sqrt{5 / 3}$ for $Y$ and $g^{\prime}$, respectively.
} 


\begin{tabular}{|l|l|l|l|l|l|l|l|l|l|l|l|l|l|l|}
\hline & \multicolumn{3}{|c|}{ Scenario-I } & \multicolumn{3}{c|}{ Scenario-II } & \multicolumn{5}{c|}{ Scenario-III (Scenario-III') } \\
\hline & \multicolumn{3}{|c|}{$\tan \theta=\sqrt{3 / 5}, \delta=-1 / 3$} & \multicolumn{3}{c|}{$\tan \theta=\sqrt{15}, \delta=-\sqrt{10} / 3$} & \multicolumn{5}{c|}{$\tan \theta=\sqrt{5 / 3}, \delta=-\sqrt{5 / 12}$} \\
\hline & $2 \sqrt{6} Q_{\psi}$ & $2 \sqrt{10} Q_{\chi}$ & $\sqrt{15} Q^{\prime}$ & $\sqrt{15} \bar{Q}$ & $2 \sqrt{6} Q_{\psi}$ & $2 \sqrt{10} Q_{\chi}$ & $\sqrt{6} Q^{\prime}$ & $\sqrt{6} \bar{Q}$ & $2 \sqrt{6} Q_{\psi}$ & $2 \sqrt{10} Q_{\chi}$ & $Q^{\prime}$ & $\bar{Q}$ \\
\hline$Q$ & 1 & -1 & 1 & $5 / 6$ & 1 & -1 & $1 / 2$ & $1 / 6$ & 1 & -1 & $1 / 4$ & $1 / 6$ \\
$u^{c}$ & 1 & -1 & 1 & $5 / 3$ & 1 & 3 & -1 & $1 / 3$ & 1 & 3 & $-1 / 4$ & $1 / 12$ \\
$d^{c}$ & 1 & 3 & $-1 / 2$ & $-5 / 6$ & 1 & -1 & $1 / 2$ & $-1 / 6$ & $1(1)$ & $-1(3)$ & $1 / 4(-1 / 4)$ & $1 / 12(-5 / 12)$ \\
$h^{c}$ & -2 & -2 & $-1 / 2$ & $-5 / 6$ & -2 & -2 & $1 / 2$ & $-1 / 6$ & $1(1)$ & $3(-1)$ & $-1 / 4(1 / 4)$ & $-5 / 12(1 / 12)$ \\
$L$ & 1 & 3 & $-1 / 2$ & 0 & 1 & 3 & -1 & 0 & 1 & 3 & $-1 / 4$ & 0 \\
$e^{c}$ & 1 & -1 & 1 & 0 & 1 & -5 & 2 & 0 & 4 & 0 & $1 / 2$ & 0 \\
\hline
\end{tabular}

\begin{tabular}{|l|l|l|l|l|l|l|l|l|l|l|l|l|l|}
\hline & \multicolumn{4}{|c|}{ Scenario-IV } & \multicolumn{4}{c|}{ Scenario-V (Scenario-V') } & \multicolumn{3}{c|}{ Scenario-VI } \\
\hline & \multicolumn{3}{|c|}{$\tan \theta=\sqrt{3 / 5}, \delta=-1 / 3$} & \multicolumn{3}{c|}{$\tan \theta=\sqrt{5 / 27}, \delta=-\sqrt{5 / 12}$} & \multicolumn{3}{c|}{$\tan \theta=0, \delta=-\sqrt{10} / 3$} \\
\hline & $2 \sqrt{6} Q_{\psi}$ & $2 \sqrt{10} Q_{\chi}$ & $\sqrt{15} Q^{\prime}$ & $\sqrt{15} \bar{Q}$ & $2 \sqrt{6} Q_{\psi}$ & $2 \sqrt{10} Q_{\chi}$ & $Q^{\prime}$ & $\bar{Q}$ & $2 \sqrt{6} Q_{\psi}$ & $2 \sqrt{10} Q_{\chi}$ & $\sqrt{6} Q^{\prime}$ & $\sqrt{6} \bar{Q}$ \\
\hline$Q$ & 1 & -1 & 1 & $5 / 6$ & 1 & -1 & $1 / 4$ & $1 / 6$ & 1 & -1 & $1 / 2$ & $1 / 6$ \\
$u^{c}$ & 1 & -1 & 1 & $5 / 3$ & 1 & 3 & 0 & $1 / 3$ & 1 & 3 & $1 / 2$ & $11 / 6$ \\
$d^{c}$ & 1 & 3 & $-1 / 2$ & $-5 / 6$ & $1(-2)$ & $-1(-2)$ & $1 / 4(-1 / 4)$ & $1 / 12(-5 / 12)$ & 1 & -1 & $1 / 2$ & $-1 / 6$ \\
$h^{c}$ & -2 & -2 & $-1 / 2$ & $-5 / 6$ & $-2(1)$ & $-2(-1)$ & $-1 / 4(1 / 4)$ & $-5 / 12(1 / 12)$ & 1 & 3 & $1 / 2$ & $-1 / 6$ \\
$L$ & -2 & -2 & $-1 / 2$ & 0 & -2 & -2 & $-1 / 4$ & 0 & -2 & -2 & -1 & 0 \\
$e^{c}$ & 1 & -1 & 1 & 0 & 1 & -5 & $1 / 2$ & 0 & 4 & 0 & 2 & 0 \\
\hline
\end{tabular}

Table 4. U(1) charges of six leptophobic scenarios in the $E_{6}$ GUT model. Fields with a superscript $c$ denote the corresponding charge-conjugated fields.

$g^{\prime} \tilde{g}_{Z^{\prime}} /\left(24 \pi^{2}\right) \sum Q^{\prime} Y \ln \left(|q|^{2} / M_{G U T}^{2}\right)$, where the sum is taken over the matter contents inside the loop, and $|q|$ is the electroweak scale and $M_{G U T}$ is the GUT scale. Then it would be possible to obtain the kinetic mixing of $O(0.1)$ to $O(1)$ required to realize leptophobia. Through a non-unitary transformation,

$$
\left(\begin{array}{c}
\tilde{B}_{\mu} \\
\tilde{Z}^{\prime}{ }_{\mu}
\end{array}\right)=\left(\begin{array}{cc}
1 & -\tan \chi \\
0 & \sec \chi
\end{array}\right)\left(\begin{array}{c}
B_{\mu} \\
Z_{\mu}^{\prime}
\end{array}\right),
$$

the gauge fields $\tilde{Z}_{\mu}^{\prime}$ and $\tilde{B}_{\mu}$ are diagonalized to the fields $Z_{\mu}^{\prime}$ and $B_{\mu}$ of mass eigenstates. The interaction terms are then rewritten as

$$
\mathcal{L}_{\text {int }}=-\bar{\psi} \gamma^{\mu}\left(g T^{a} W_{\mu}^{a}+g^{\prime} Y B_{\mu}+g_{Z^{\prime}} \bar{Q} Z_{\mu}^{\prime}\right) \psi,
$$

where $g_{Z^{\prime}} \equiv \tilde{g}_{Z^{\prime}} / \cos \chi$, and the $Z^{\prime}$ charge $\bar{Q}$ for a fermion field $f$ is

$$
\bar{Q}(f) \equiv Q^{\prime}(f)+\sqrt{\frac{3}{5}} \delta Y, \text { with } \delta \equiv-\sqrt{\frac{5}{3}} \frac{g^{\prime}}{g_{Z^{\prime}}} \tan \chi .
$$

As is evident from eq. (A.7), all $\bar{Q}(f)$ charges are determined by two unknown parameters; i.e., $\theta$ and $\delta$. This implies that once we fix two of $\bar{Q}(f)$ charges, all the other $\bar{Q}(f)$ charges are uniquely determined as well. We utilize this feature to find scenarios with a leptophobic $Z^{\prime}$; that is,

$$
\bar{Q}(L)=Q^{\prime}(L)-\frac{1}{2} \delta=0, \quad \bar{Q}\left(e^{c}\right)=Q^{\prime}\left(e^{c}\right)+\delta=0 .
$$

These two equations are solved to render

$$
\tan \theta=\frac{2 Q_{\psi}(L)+Q_{\psi}\left(e^{c}\right)}{2 Q_{\chi}(L)+Q_{\chi}\left(e^{c}\right)}, \quad \delta=-Q^{\prime}\left(e^{c}\right)\left(=2 Q^{\prime}(L)\right) .
$$


There are then six ways to embed the SM fermions along with exotic fermion denoted by $h$ into the $\mathbf{2 7}$ representation of $E_{6}$. In table 4, U(1) charges of the fermions are listed for the six scenarios, following the convention of ref. [41]. In this table, only $h$ is a non-SM fermion whose SM gauge quantum numbers are the same as those of $d$. Thus, one can interchange the $\mathrm{U}(1)_{\psi}$ and $\mathrm{U}(1)_{\chi}$ charges of $d$ with those of $h$. After the interchange, the $Q^{\prime}$ and $\bar{Q}$ charges of $d$ and $h$ are different from the original ones only in Scenario-III and Scenario-V. We denote the two new scenarios by Scenario-III' and Scenario-V', respectively.

Open Access. This article is distributed under the terms of the Creative Commons Attribution License (CC-BY 4.0), which permits any use, distribution and reproduction in any medium, provided the original author(s) and source are credited.

\section{References}

[1] ATLAS collaboration, Observation of a new particle in the search for the standard model Higgs boson with the ATLAS detector at the LHC, Phys. Lett. B 716 (2012) 1 [arXiv: 1207.7214] [INSPIRE].

[2] CMS collaboration, Observation of a new boson at a mass of $125 \mathrm{GeV}$ with the CMS experiment at the LHC, Phys. Lett. B $\mathbf{7 1 6}$ (2012) 30 [arXiv:1207.7235] [INSPIRE].

[3] E. Ma, Particle dichotomy and left-right decomposition of $E_{6}$ superstring models, Phys. Rev. D 36 (1987) 274 [InSPIRE].

[4] K. Babu, X.-G. He and E. Ma, New supersymmetric left-right gauge model: Higgs boson structure and neutral current analysis, Phys. Rev. D 36 (1987) 878 [INSPIRE].

[5] F. Zwirner, Phenomenological aspects of $E_{6}$ superstring-inspired models, Int. J. Mod. Phys. A 3 (1988) 49 [INSPIRE].

[6] J.L. Hewett and T.G. Rizzo, Low-energy phenomenology of superstring inspired $E_{6}$ models, Phys. Rept. 183 (1989) 193 [INSPIRE].

[7] Y. Daikoku and H. Okada, $S_{4} \times Z_{2}$ flavor symmetry in supersymmetric extra $\mathrm{U}(1)$ model, Phys. Rev. D 82 (2010) 033007 [arXiv:0910.3370] [InSPIRE].

[8] L.M. Krauss and F. Wilczek, Discrete gauge symmetry in continuum theories, Phys. Rev. Lett. 62 (1989) 1221 [INSPIRE].

[9] J. Kubo and D. Suematsu, Neutrino masses and CDM in a non-supersymmetric model, Phys. Lett. B 643 (2006) 336 [hep-ph/0610006] [INSPIRE].

[10] S. Kanemura, O. Seto and T. Shimomura, Masses of dark matter and neutrino from TeV scale spontaneous $U(1)_{B-L}$ breaking, Phys. Rev. D 84 (2011) 016004 [arXiv:1101.5713] [INSPIRE].

[11] S. Kanemura, T. Nabeshima and H. Sugiyama, TeV-scale seesaw with loop-induced Dirac mass term and dark matter from $U(1)_{B-L}$ gauge symmetry breaking, Phys. Rev. D 85 (2012) 033004 [arXiv:1111.0599] [INSPIRE].

[12] C.-W. Chiang, T. Nomura and J. Tandean, Dark matter and Higgs boson in a model with discrete gauge symmetry, Phys. Rev. D 87 (2013) 073004 [arXiv:1205.6416] [INSPIRE]. 
[13] S. Kanemura, T. Nabeshima and H. Sugiyama, Radiative type-I seesaw model with dark matter via $\mathrm{U}(1)_{B-L}$ gauge symmetry breaking at future linear colliders, Phys. Rev. D 87 (2013) 015009 [arXiv:1207.7061] [INSPIRE].

[14] Y. Daikoku, H. Okada and T. Toma, Two component dark matters in $S_{4} \times Z_{2}$ flavor symmetric extra U(1) model, Prog. Theor. Phys. 126 (2011) 855 [arXiv:1106.4717] [INSPIRE].

[15] Y. Daikoku and H. Okada, Phenomenology of $S_{4}$ flavor symmetric extra U(1) model, Phys. Rev. D 88 (2013) 015034 [arXiv:1303.7056] [inSPIRE].

[16] S. Ham, E. Yoo and S. Oh, Explicit CP-violation in a MSSM with an extra U(1)', Phys. Rev. D 76 (2007) 015004 [hep-ph/0703041] [INSPIRE].

[17] S. Ham, E. Yoo and S. OH, Electroweak phase transitions in the MSSM with an extra U(1)', Phys. Rev. D 76 (2007) 075011 [arXiv:0704.0328] [InSPIRE].

[18] C.-W. Chiang and E. Senaha, Electroweak phase transitions in the secluded U(1)'-extended MSSM, JHEP 06 (2010) 030 [arXiv:0912.5069] [INSPIRE].

[19] A. Ahriche and S. Nasri, Electroweak phase transition in the U(1)'-MSSM, Phys. Rev. D 83 (2011) 045032 [arXiv: 1008.3106] [INSPIRE].

[20] E. Senaha, $Z^{\prime}$-ino-driven electroweak baryogenesis in the UMSSM, Phys. Rev. D 88 (2013) 055014 [arXiv: 1308.3389] [INSPIRE].

[21] V. Kuzmin, V. Rubakov and M. Shaposhnikov, On the anomalous electroweak baryon number nonconservation in the early Universe, Phys. Lett. B 155 (1985) 36 [INSPIRE].

[22] P. Langacker, The physics of heavy Z' gauge bosons, Rev. Mod. Phys. 81 (2009) 1199 [arXiv:0801.1345] [INSPIRE].

[23] P. Langacker and M. Plümacher, Flavor changing effects in theories with a heavy $Z^{\prime}$ boson with family nonuniversal couplings, Phys. Rev. D 62 (2000) 013006 [hep-ph/0001204] [INSPIRE].

[24] ATLAS collaboration, Search for high-mass dilepton resonances in $20 \mathrm{fb}^{-1}$ of pp collisions at $\sqrt{s}=8 \mathrm{TeV}$ with the ATLAS experiment, ATLAS-CONF-2013-017 (2013).

[25] ATLAS collaboration, A search for high-mass ditau resonances decaying in the fully hadronic final state in pp collisions at $\sqrt{s}=8 \mathrm{TeV}$ with the ATLAS detector, ATLAS-CONF-2013-066 (2013).

[26] K. Babu, C.F. Kolda and J. March-Russell, Leptophobic U(1) s and the R(b)-R(c) crisis, Phys. Rev. D 54 (1996) 4635 [hep-ph/9603212] [INSPIRE].

[27] K. Babu, C.F. Kolda and J. March-Russell, Implications of generalized Z-Z' mixing, Phys. Rev. D 57 (1998) 6788 [hep-ph/9710441] [INSPIRE].

[28] V.D. Barger, K.-m. Cheung and P. Langacker, Baryonic $Z^{\prime}$ connection of LEP R(b,c) data with Tevatron $(W, Z, \gamma) b \bar{b}$ events, Phys. Lett. B 381 (1996) 226 [hep-ph/9604298] [INSPIRE].

[29] M. Heyssler, A leptophobic massive vector boson at the Tevatron and the LHC, Phys. Rev. D 54 (1996) 5845 [hep-ph/9605403] [INSPIRE].

[30] F. del Aguila and J. Aguilar-Saavedra, Like-sign dilepton signals from a leptophobic $Z^{\prime}$ boson, JHEP 11 (2007) 072 [arXiv:0705.4117] [INSPIRE].

[31] V. Barger, P. Langacker and H.-S. Lee, Six-lepton $Z^{\prime}$ resonance at the LHC, Phys. Rev. Lett. 103 (2009) 251802 [arXiv:0909.2641] [INSPIRE]. 
[32] H. An, R. Huo and L.-T. Wang, Searching for low mass dark portal at the LHC, Phys. Dark Univ. 2 (2013) 50 [arXiv:1212.2221] [INSPIRE].

[33] A. Alves, S. Profumo and F.S. Queiroz, The dark $Z^{\prime}$ portal: direct, indirect and collider searches, JHEP 04 (2014) 063 [arXiv:1312.5281] [INSPIRE].

[34] M.R. Buckley, D. Hooper, J. Kopp and E. Neil, Light $Z^{\prime}$ bosons at the Tevatron, Phys. Rev. D 83 (2011) 115013 [arXiv:1103.6035] [InSPIRE].

[35] K. Cheung and J. Song, Baryonic $Z^{\prime}$ explanation for the CDF $W_{j j}$ excess, Phys. Rev. Lett. 106 (2011) 211803 [arXiv:1104.1375] [INSPIRE].

[36] K. Cheung and J. Song, The Golden Mode for a baryonic $Z^{\prime}$ boson at hadronic colliders: $p p / p \bar{p} \rightarrow W Z^{\prime} \rightarrow l \nu b \bar{b}$, Phys. Rev. D 84 (2011) 034043 [arXiv:1106.6141] [InSPIRE].

[37] Y. Umeda, G.-C. Cho and K. Hagiwara, Constraints on leptophobic Z' models from electroweak experiments, Phys. Rev. D 58 (1998) 115008 [hep-ph/9805447] [INSPIRE].

[38] G.-C. Cho, K. Hagiwara and Y. Umeda, $Z$ ' bosons in supersymmetric $E_{6}$ models confront electroweak data, Nucl. Phys. B 531 (1998) 65 [Erratum ibid. B 555 (1999) 651] [hep-ph/9805448] [INSPIRE].

[39] M.R. Buckley and M.J. Ramsey-Musolf, Precision probes of a leptophobic $Z^{\prime}$ boson, Phys. Lett. B 712 (2012) 261 [arXiv:1203.1102] [INSPIRE].

[40] M. González-Alonso and M.J. Ramsey-Musolf, Leptophobic $Z^{\prime}$ boson and parity-violating eD scattering, Phys. Rev. D 87 (2013) 055013 [arXiv:1211.4581] [INSPIRE].

[41] K. Leroux and D. London, Flavor changing neutral currents and leptophobic $Z^{\prime}$ gauge bosons, Phys. Lett. B 526 (2002) 97 [hep-ph/0111246] [INSPIRE].

[42] T.G. Rizzo, Gauge kinetic mixing and leptophobic $Z^{\prime}$ in $E_{6}$ and $\mathrm{SO}(10)$, Phys. Rev. D 59 (1998) 015020 [hep-ph/9806397] [INSPIRE].

[43] A. Pukhov, CalcHEP 2.3: MSSM, structure functions, event generation, batchs and generation of matrix elements for other packages, hep-ph/0412191 [INSPIRE].

[44] CMS collaboration, Search for $t \bar{t}$ resonances in semileptonic final state, CMS-PAS-B2G-12-006 (2013).

[45] B.A. Dobrescu and F. Yu, Coupling-mass mapping of dijet peak searches, Phys. Rev. D 88 (2013) 035021 [arXiv: 1306.2629] [InSPIRE].

[46] O. Domenech, A. Pomarol and J. Serra, Probing the SM with dijets at the LHC, Phys. Rev. D 85 (2012) 074030 [arXiv:1201.6510] [InSPIRE].

[47] ATLAS collaboration, Search for new physics in dijet mass and angular distributions in pp collisions at $\sqrt{s}=7 \mathrm{TeV}$ Measured with the ATLAS detector, New J. Phys. 13 (2011) 053044 [arXiv:1103.3864] [INSPIRE].

[48] ATLAS collaboration, ATLAS search for new phenomena in dijet mass and angular distributions using pp collisions at $\sqrt{s}=7 \mathrm{TeV}$, JHEP 01 (2013) 029 [arXiv:1210.1718] [INSPIRE].

[49] CMS collaboration, Measurement of the production cross section for $a W$ boson and two $b$ jets in pp collisions at $\sqrt{s}=7 \mathrm{TeV}$, arXiv:1312.6608 [INSPIRE].

[50] CMS collaboration, Measurement of the production cross sections for a $Z$ boson and one or more $b$ jets in pp collisions at $\sqrt{s}=7 \mathrm{TeV}$, arXiv:1402.1521 [INSPIRE]. 
[51] J. Alwall, M. Herquet, F. Maltoni, O. Mattelaer and T. Stelzer, MadGraph 5: going beyond, JHEP 06 (2011) 128 [arXiv:1106.0522] [INSPIRE].

[52] CMS collaboration, Study of the dijet mass spectrum in $p p \rightarrow W+$ jets events at $\sqrt{s}=7$ TeV, Phys. Rev. Lett. 109 (2012) 251801 [arXiv: 1208.3477] [INSPIRE].

[53] UA2 collaboration, J. Alitti et al., A search for new intermediate vector mesons and excited quarks decaying to two jets at the CERN p̄p collider, Nucl. Phys. B 400 (1993) 3 [INSPIRE].

[54] T. Sjöstrand, S. Mrenna and P.Z. Skands, PYTHIA 6.4 physics and manual, JHEP 05 (2006) 026 [hep-ph/0603175] [INSPIRE].

[55] http://www.physics.ucdavis.edu/conway/research/software/pgs/pgs4-general.htm.

[56] CMS collaboration, CMS technical design report, volume II: physics performance, J. Phys. G 34 (2007) 995 [inSPIRE].

[57] V. Skalozub and I. Kucher, Observables for model-independent detections of $Z^{\prime}$ boson at the ILC, arXiv: 1402.1187 [INSPIRE].

[58] C.W. Chiang, T. Nomura and K. Yagyu, work in preparation.

[59] B. Holdom, Two U(1)'s and epsilon charge shifts, Phys. Lett. B 166 (1986) 196 [InSPIRE].

[60] K.R. Dienes, C.F. Kolda and J. March-Russell, Kinetic mixing and the supersymmetric gauge hierarchy, Nucl. Phys. B 492 (1997) 104 [hep-ph/9610479] [INSPIRE]. 